receiving either Rha-DOX or glycosylated rhamnosidase alone.

LEAPT achieves higher concentrations of active drug at the target site through the use of engineered glycosylated enzymes than delivery of drug alone. Assuming that suitable receptors can be identified, delivery to other cell types is possible simply by changing the cognate sugar attached to the enzyme. The use of macrophage-associated carbohydrate receptors to treat macrophageassociated diseases such as lysosomal storage diseases or viral infections could benefit from this approach.

Melanie Brazil

(D) References and links ORIGINAL RESEARCh PAPER Robinson, M. A. et al. LEAPT: lectin-directed enzyme-activated prodrug therapy. Proc. Natl Acad. Sci. 24 Sep 2004 (doi:10.1073/pnas.0303574101) FURTHER READING

Duncan, R. The dawning era of polyme therapeutics. Nature Rev. Drug Discov. 2 347-360 (2003) | Prausnitz, M. R., Mitrogotri, S. \& Langer, R. Current status and future potential of transdermal drug delivery. Nature Rev. Drug Discov. 3, 115-124 (2004)

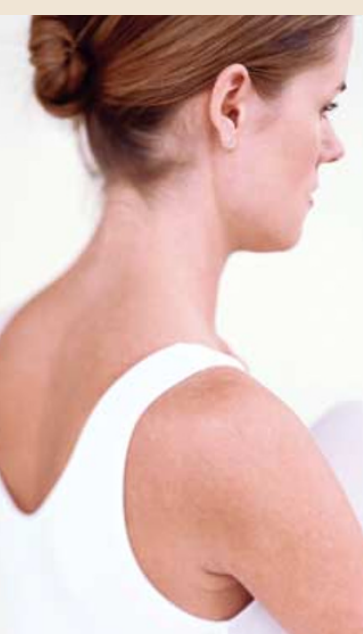

\section{Screen if you want to go faster!}

Historically, screening methods and diagnostics have been limited by the requirement to either directly label a chemical compound, biochemical molecule or cell using methods such as fluorescence or radioactivity; or to incorporate labelling stages, such as incubation with an antibody followed by a series of washes. These techniques, although sensitive and amenable to configuration in a high-throughput format, are expensive and there is the risk that altering the target molecule could affect its activity. Much effort has therefore been invested in developing label-free screening technologies, particularly as we enter an era in which faster methods for screening a glut of genomic targets are required. In the September issue of the Journal of Biomolecular Screening, four papers highlight novel label-free detection methods.

In the first paper, Huff et al. describe the use of the atomic force microscope (AFM) for the detection of proteins and pathogens, which can be coupled with protein nano-arrays. AFM uses a sharp, micron-scale tip to scan and amplify the topographical features of proteins and can therefore detect molecules bound to proteins by detecting nanometre changes in height. Coating the AFM tip with biological molecules provides the opportunity to bind specific proteins and can allow measurements of intermolecular binding constants, enabling the detection of single antibody-antigen interactions. The authors have developed a label-free, multiplexed, chip-based detection system that can directly determine the amount of virus particles in a sample.

In the second paper, Chambrone et al. describe how the measurement of bioimpedance can be used for the label-free detection and characterization of a cellular response to ligand-receptor binding. The method works by applying alternating voltages at set frequencies to cellular components (such as membranes) and intact cells, and monitors the resulting currents generated from the dipoles within the sample (reflected currents). The technique can detect different patterns of bioimpedance according to the particular receptor pathway being studied and the cell line being used, and could be applied in hit confirmation and pharmacological evaluation of receptor activity in response to an agonist or antagonist.

The third paper addresses one of the hardest challenges for screening technologies: detecting protein-protein interactions. The Biomolecular Interaction Detection (BIND) system described

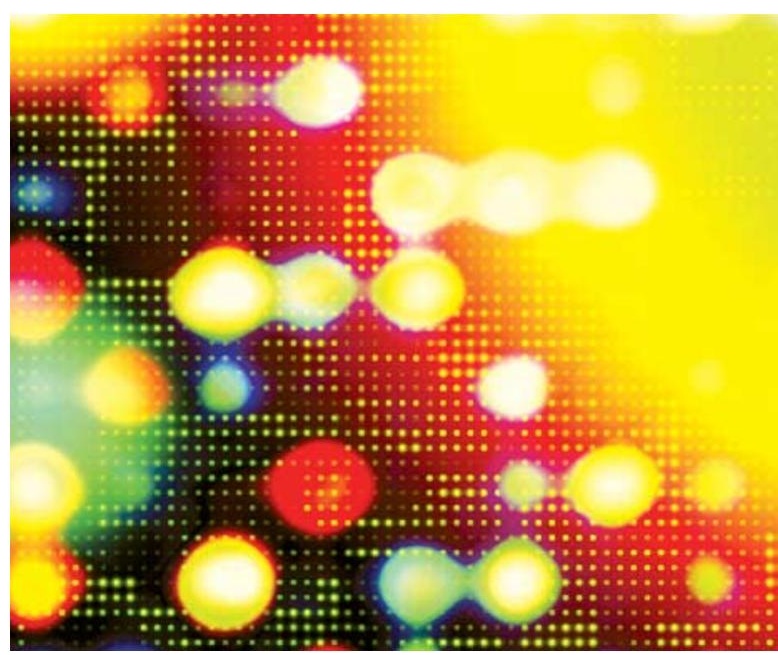

by Cunningham et al. is able to characterize these interactions, as well as small-molecule-protein interactions, protein-cell interactions and cell toxicity, in a high-throughput manner. BIND is a guided-mode resonant filter (GMRF) biosensor, which essentially means that on illumination with white light, the biosensor reflects only a narrow band of wavelengths. This narrow band can be optimized to provide a wavelength that is particularly sensitive to modulations that occur when a biochemical material is placed on its surface. The simple format of the BIND assay means that it can be manufactured as continuous sheets of plastic that can be tailored to suit 96-, 384- and 1,536-well assay formats.

A fourth paper by Zehender et al. describes a method for screening orphan or intractable targets, which is often hindered by stringent assay conditions that can miss low-affinity binders. The SpeedScreen comprises a homogenous solution-based affinity selection technique, followed by size-exclusion chromatography and microbore-liquid-chromatography/electrosprayionization mass spectrometry. The solutionbased affinity-selection method enables the detection of low-affinity binders that are often removed during the washing stages of methods in which the target is immobilized.

Joanna Owens

(0) References and links

ORIGINAL RESEARCH PAPERS Huff, J. L. et al. Label-free protein and pathogen detection using the atomic force microscope. J. Biomol. Screen. 9, 467-480 (2004) | Chambrone, G. J. et al. Cellular dielectric spectroscopy: a powerful new approach to label-free cellular analysis. J. Biomol. Screen. 9, 491-497 (2004) | Cunningham, B. T. et al. Labelfree assays on the BIND system. J. Biomol. Screen. 9, 481-490 (2004) Zehender, $\mathrm{H}$. et al. SpeedScreen: the 'missing link' between genomics and lead discovery. J. Biomol. Screen. 9, 498-505 (2004). 